\title{
The Cardioband transcatheter direct mitral valve annuloplasty system
}

\author{
Francesco Maisano*, MD, FESC; Maurizio Taramasso, MD \\ Division of Cardiovascular Surgery, Zurich University Hospital, Zurich, Switzerland
}

\section{Device description}

Name and manufacturer: The Cardioband System; Valtech Cardio Ltd., Or Yehuda, Israel (Figure 1A).

Approval status: CE mark expected in 2015.

Implant technology: The Cardioband implant comprises three components: 1) a standard polyester fabric sleeve; 2) multiple stainless steel anchors; 3) an adjustment mechanism. The prosthesis is attached to the posterior annulus, trigone to trigone, via subsequent anchors. The adjustment mechanism is embedded inside the sleeve and after full deployment of the implant allows a homogeneous circumferential annular cinching (Figure 1B).

Delivery system: The Cardioband transfemoral delivery system consists of three components: 1) the implant delivery system features five degrees of control for continuous navigation across the mitral annulus (Figure 1C); 2) the anchor driver is used for anchor insertion with one-to-one torque transfer; 3) the size adjustment tool (Figure 1D), together with the adjustment mechanism inside the implant, provides a homogeneous stepwise cinching in which adequate remodelling is confirmed by echo on beating heart.

Delivery method: Transfemoral transseptal venous access.

Implant sizes: The Cardioband implant is available in six sizes to cover the different annulus sizes.

\section{Procedural details}

The procedure is stepwise, straightforward and reproducible, in which each step is reversible, resulting in an enhanced level of safety and control throughout. 1) Access: the device is introduced through the femoral vein to the right atrium and then through the fossa ovalis to the left atrium using a transseptal approach (Figure 2A). 2) Implant deployment: the implant delivery system is steered to reach any point on the mitral annulus. Subsequent anchors are deployed as the tip is navigated along the posterior annulus, as an annuloplasty ring would be sutured during an open heart procedure (Figure 2B, Figure 2C). 3) Implant adjustment, annular remodelling and MR reduction: after completion of implant deployment, the size adjustment tool (SAT) is introduced over a wire. By turning a knob, the implant is adjusted bi-directionally to reshape the annulus (Figure 2D), confirming MR reduction by echocardiography under beating heart.

\section{Clinical data}

The first-in-man (FIM) multicentre clinical study is currently ongoing, evaluating the feasibility, safety and performance of the Cardioband transcatheter adjustable mitral annuloplasty system in patients with functional mitral regurgitation (FMR). Between February 2013 and July 2015, 45 high-risk patients with significant FMR were enrolled at six sites in Europe. After a Heart Team evaluation, all patients were screened by echocardiography to assess eligibility.

Echocardiographic data were analysed by an independent core lab (Baylor Research Institute; Paul A. Grayburn, MD, USA). Mean age was $71 \pm 8$ years; thirty-four patients were

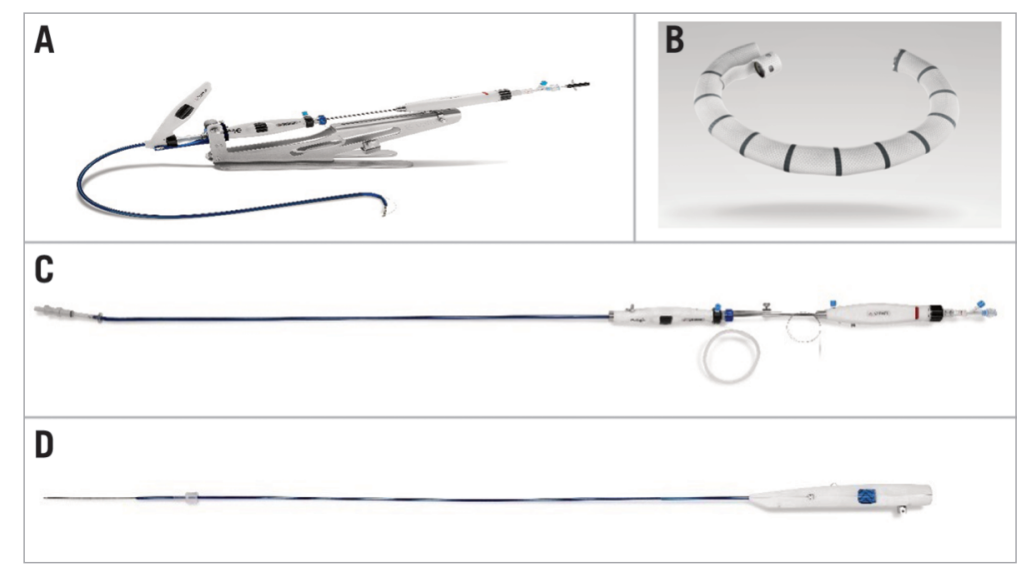

Figure 1. The Cardioband System. The Cardioband System (A) is composed of the implant (B), the delivery system (C) and the size adjustment tool (D).

*Corresponding author: Division of Cardiovascular Surgery, Zurich University Hospital, Rämistrasse 100, CH-8091 Zurich, Switzerland.E-mail: francesco.maisano@usz.ch 

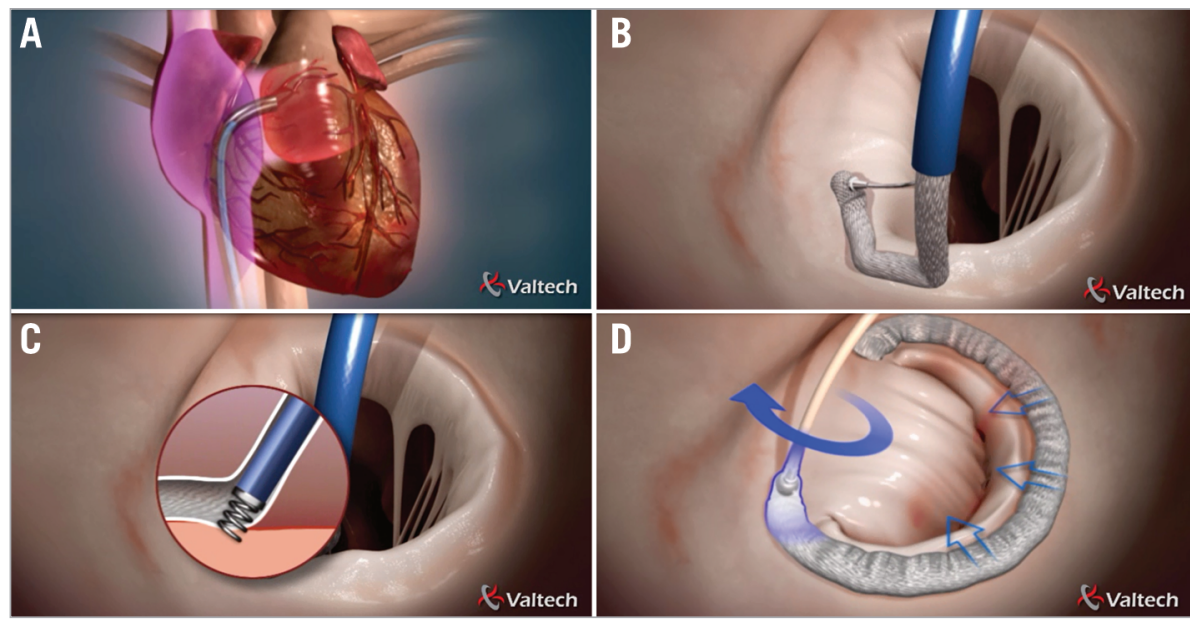

Figure 2. The Cardioband procedure. The procedure begins with transseptal access to the left atrium (A), then implant deployment (B), multiple anchor implantation $(C)$, and size adjustment with mitral annulus remodelling $(D)$.

male (76\%). Mean logistic EuroSCORE was $17 \pm 12 \%$. At baseline, $87 \%$ of patients were in NYHA Class III-IV with mean EF of $32 \pm 11 \%(15 \%-59 \%)$. Device implantation was feasible in all patients $(100 \%)$. MR reduction to $\leq 1+$ was achieved in $84 \%$ of the patients (38/45) intra-procedure. After cinching of the device, remodelling of the mitral annulus with an average of $20 \%$ reduction of the septolateral diameter was observed (from $36 \pm 5 \mathrm{~mm}$ to $29 \pm 6 \mathrm{~mm} ; \mathrm{p}<0.01)$. Thirty-day mortality was $4.4 \%$ (adjudicated as unrelated to the device). At six-month followup, $82 \%$ of patients were in NYHA Class I-II $(n=22)$ with significant improvement in quality of life (MLWHFQ from 38 to $18 ; \mathrm{p}<0.05 ; \mathrm{n}=21)$ and $86 \%$ of patients had $\mathrm{MR} \leq 2+(\mathrm{n}=22)$. At 12 -month follow-up, $94 \%$ of patients had MR $\leq 2+(n=17)$, and $68 \%$ of patients were in NYHA Class I-II $(n=18, p<0.05)$, confirming a positive trend in quality of life (MLWHFQ from 35 to $19 ; \mathrm{p}<0.05 ; \mathrm{n}=16)$. These results show the feasibility of transseptal direct annuloplasty with the Cardioband system, with a sustained safety profile, similar to other transcatheter mitral procedures. Effective reduction in MR severity is observed in most patients related to a significant septolateral dimension reduction. MR reduction is stable and consistent up to 12 months, with significant clinical benefit.

\section{Ongoing studies}

The Cardioband clinical study (NCT01841554) is currently ongoing.

\section{Unique features}

The Cardioband System features a unique segmental deployment that conforms easily to each patient's specific annular geometry.

The venous access, combined with supra-annular fixation, as in surgery, and the stepwise procedure, which is reversible, guarantee an enhanced level of safety and control throughout the procedure ${ }^{1-3}$.
The implant enables significant reduction of annular dimensions, with preservation of the native anatomy.

\section{Potential improvements}

Cardioband, while reshaping and stabilising the mitral annulus, can be used in combination with other mitral valve transcatheter therapies, such as edge-to-edge repair or chordal replacement, to provide a surgical-like treatment to most types of mitral valve insufficiency ${ }^{4}$.

\section{Conflict of interest statement}

F. Maisano is a consultant for Abbott Vascular, Valtech Cardio, Medtronic, Edwards Lifesciences, St. Jude, and Apica, is co-founder of 4Tech, and receives royalties from Edwards Lifesciences. M. Taramasso has no conflicts of interest to declare.

\section{References}

1. Maisano F, Reser D, Pavicevic J, Guidotti A, Denti P, Taramasso M, Addis A, Cesarovic N, Emmert MY, Nietlispach F, Swain J, Falk V, Leon M. A translational "humanised" porcine model for transcatheter mitral valve interventions: the neo inferior vena cava approach. EuroIntervention. 2015;11:92-5.

2. Sündermann SH, Gordic S, Manka R, Cesarovic N, Falk V, Maisano F, Alkadhi H. Computed tomography for planning and postoperative imaging of transvenous mitral annuloplasty: first experience in an animal model. Int J Cardiovasc Imaging. 2015;31:135-42.

3. Maisano F, La Canna G, Latib A, Denti P, Taramasso M, Kuck KH, Colombo A, Alfieri O, Guidotti A, Messika-Zeitoun D, Vahanian A. First-in-man transseptal implantation of a "surgicallike" mitral valve annuloplasty device for functional mitral regurgitation. JACC Cardiovasc Interv. 2014;7:1326-8.

4. Maisano F, Kuck KH. Transcatheter mitral direct annuloplasty: state of the art. Minerva Cardioangiol. 2014;62:251-9. 